Itinéraires Itinéraires

Littérature, textes, cultures

\title{
La « modernité viennoise »: de la réception du naturalisme à une " mystique des nerfs »
}

\section{Karl Zieger}

\section{OpenEdition}

\section{Journals}

Édition électronique

URL : http://journals.openedition.org/itineraires/518

DOI : 10.4000/itineraires.518

ISSN : 2427-920X

Éditeur

Pléiade

\section{Édition imprimée}

Date de publication : 1 novembre 2009

Pagination : 135-149

ISBN : 978-2-296-10115-9

ISSN : 2100-1340

\section{Référence électronique}

Karl Zieger, «La «modernité viennoise »: de la réception du naturalisme à une « mystique des nerfs » », Itinéraires [En ligne], 2009-3 | 2009, mis en ligne le 25 juin 2014, consulté le 20 avril 2019. URL : http://journals.openedition.org/itineraires/518 ; DOI : 10.4000/itineraires.518

\section{(C) $(\oplus \Theta$}

Itinéraires est mis à disposition selon les termes de la licence Creative Commons Attribution - Pas d'Utilisation Commerciale - Pas de Modification 4.0 International. 


\title{
La « modernité viennoise » : de la réception du naturalisme à une « mystique des nerfs »
}

\begin{abstract}
The turn of the nineteenth century is probably the best known period of Austrian literature. Within the span of a few years, such writers as Arthur Schnitzler, Hugo von Hofmannsthal, Richard Beer-Hofmann and a few others evolved from bourgeois realism to fin-de-siècle estheticism, incorporating such movements as naturalism, impressionism, symbolism and decadence. The group they formed, "The Young Vienna", has become emblematic for modernity. Through the analysis of the journal Moderne Dichtung/Moderne Runcschau, the present article first examines the role of naturalism as a starting element of Vienna modernity. It then focuses on Hermann Bahr who, with his concept of "going beyond naturalism" provided the writers of "The Young Vienna" with the image that allowed them to be identified far beyond the borders of their country.
\end{abstract}

Keywords : Vienna modernity, Naturalism, Symbolism, Hermann Bahr, Moderne Dichtung / Moderne Rundschau

Mots clés : modernité viennoise, naturalisme, symbolisme, Hermann Bahr, Moderne Dichtung / Moderne Rundschau

L'une des périodes de la littérature autrichienne les mieux connues à l'étranger - et notamment en France - est celle du tournant du XIX ${ }^{e}$ au $X^{e}$ siècle. Les dernières décennies de l'Empire austro-hongrois ont effectivement connu une effervescence culturelle assez exceptionnelle dans beaucoup de domaines (littérature, peinture, musique, philosophie, psychologie) et les travaux sur les représentants de ce que l'on désigne généralement par le terme de Wiener Moderne («modernité viennoise ») sont désormais nombreux ${ }^{1}$.

1. Une synthèse utile de l'état de la recherche se trouve dans Dagmar Lorenz, Wiener Moderne, Stuttgart, Metzler, 2007 ( $2^{\mathrm{e}}$ édition revue et actualisée). En France, l'exposition Vienne 1880-1938 au Centre Pompidou en 1986 a suscité de nombreux travaux sur cette 
Dans le domaine littéraire, ce sont les écrivains de la génération née entre 1860 et 1875, regroupés sous l'étiquette de Das junge Wien (la « Jeune Vienne »), les Hofmannsthal, Schnitzler, Beer-Hofmann, Peter Altenberg et, dans leur sillage, Stefan Zweig qui sont les plus connus et les plus étudiés. Dans l'existence de ce groupe un rôle particulier revient à Hermann Bahr : celui du grand communicateur, du promoteur du groupe, rôle souvent controversé, mais important à cause de l'activité journalistique qu'il a déployée.

Ce groupe a été connu en France dès le tournant du XIX ${ }^{\mathrm{e}}$ au $\mathrm{XX}^{\mathrm{e}}$ siècle et la critique lui a attribué des caractéristiques comme « sensibilité », « raffinement» et « délicatesse ${ }^{2}$ », mais aussi un manque de profondeur et d'idées ${ }^{3}$. Aujourd'hui on caractérise cette " modernité viennoise » par son goût pour l'individu(el) et le subjectif, par la crise du moi, la crise de l'identité, la crise du langage et par la subjectivité, l'introspection, l'intérêt pour la psychologie, les états d'âme... Ces éléments sont devenus emblématiques pour la littérature autrichienne de cette époque. Certains historiens de la littérature estiment même que la «modernité viennoise » a fortement marqué la « modernité européenne » et la « culture fin de siècle ${ }^{4}$ ». Si tel est le cas, elle n'a cependant fait que rendre ce qu'elle avait reçu auparavant, car la particularité de sa genèse réside notamment dans une ouverture rapide à des tendances considérées alors comme novatrices, y compris le naturalisme. En peu de temps, au début des années 1890, les écrivains autrichiens ont parcouru le chemin qui mène d'un réalisme « bourgeois » à l'art « finde-siècle » avec toutes ses facettes; il s'agit donc d'un rattrapage rapide de l'évolution qui semble avoir touché l'Autriche... avec du retard.

\section{Un moment particulier de l'histoire culturelle de l'Autriche}

La modernité viennoise coïncide avec les dernières décennies de l'Empire des Habsbourg qui est devenu, en 1867, après la défaite contre la Prusse l'année précédente et à la suite d'un accord avec la Hongrie, la « Double Monarchie » austro-hongroise. Avec la création de l'Empire allemand en 1871 sous l'égide de la Prusse, l'Autriche-Hongrie perd progressivement son influence en Allemagne et sa politique s'oriente de plus en plus vers l'Europe centrale et vers les Balkans. Mais l'État autrichien, voire austro-

\footnotetext{
époque. Il faut citer, comme base documentaire, Michael Pollak, Vienne 1900, Paris, Gallimard/Julliard, coll. "Archives », 1984 et notamment les travaux de Jacques Le Rider, Modernité viennoise et crises de l'identité, Paris, PUF, 1990, rééd. 1994; Id., Hugo von Hofmannsthal. Historicisme et modernité, Paris, PUF, coll. " Perspectives germaniques », 1995 ; Id., Journaux intimes viennois, PUF, 2000.

2. Voir p. ex. Henri Albert, «Les Jeunes Viennois », La Revue des Revues, vol. XIII, $1^{\text {er }}$ avril 1895, p. $8-13$.

3. Voir p. ex. Maurice Muret, «Un Parisien de Vienne - M. Arthur Schnitzler », La Nouvelle Revue, $3^{\mathrm{e}}$ série, vol. IV, $1^{\mathrm{er}}$ août 1908, p. 339-354.

4. Cf. Gotthart Wunberg (dir.), Die Wiener Moderne. Literatur, Kunst und Musik zwischen 1890 und 1910, Stuttgart, Philipp Reclam jun, 1981, p. 61.
} 
hongrois, est confronté au réveil des revendications nationales dans les différents Kronländer («Pays de la Couronne »), notamment de la part des Slaves.

Une partie de la population de la monarchie danubienne, notamment de la population germanophone, regarde alors avec une certaine envie la réussite politique, économique et culturelle du nouvel Empire allemand. À ce propos, une phrase de l'Autoportrait de Hermann Bahr : "Sedan, Bismarck, Richard Wagner hatten sie, da draussen. Und was hatten wir ${ }^{5}$ ?» («Eux, là bas, avaient Sedan, Bismarck, Richard Wagner. Et nous, qu'avions-nous? ») est révélatrice.

Dans ce contexte, la recherche d'une identité autrichienne se manifeste tout naturellement. On la retrouve aussi dans le domaine littéraire, où l'on parle depuis le début du XIX ${ }^{e}$ siècle, grâce à des écrivains comme Grillparzer (1791-1872), Nestroy (1801-1862), Raimund (1790-1836) et Stifter (1805-1868), d'une littérature spécifiquement autrichienne. La volonté de se démarquer de Berlin et des mouvements littéraires de l'Empire allemand jouera de même un rôle important dans les discours critiques autour de la modernité viennoise et le groupe de la « Jeune Vienne », un groupe beaucoup moins homogène que ces discours ont voulu le faire croire.

En effet, les origines de ce qu'on appelle la «modernité viennoise » résident dans une réception presque simultanée de plusieurs mouvements qui ont déjà marqué les littératures occidentales, notamment le naturalisme, le symbolisme et la décadence française. Elle est le résultat d'influences multiples et revêt différentes facettes.

Dans cet article, nous rappellerons essentiellement deux éléments décisifs pour la constitution de la «Jeune Vienne » et de la "modernité viennoise » : l'action exercée par une revue (éphémère, certes, mais d'une influence considérable), la Moderne Dichtung/Moderne Rundschau (Poésie moderne/Revue moderne), et le rôle d'intermédiaire qu'a joué Hermann $\mathrm{Bahr}^{6}$. Ces deux facteurs nous font penser en effet que le naturalisme et la volonté de le dépasser sont à l'origine de la modernité viennoise... au même titre que l'influence exercée par les littératures française et anglaise.

\section{À l'origine de la modernité viennoise : la réception du naturalisme}

Jusqu'au début des années 1980, plusieurs historiens de la littérature germanophone ont jugé que l'on pouvait écrire l'histoire de la littérature

5. Citée d'après Gotthart Wunberg, op. cit., p. 43.

6. Nous nous basons essentiellement sur les ouvrages suivants : Hermann Bahr, Ce monsieur de Linz qui inventa Vienne. Textes de Hermann Bahr, Karl Kraus, Hugo von Hofmannsthal traduits et présentés par Jean Launay, Monaco, Éditions du Rocher, coll. « Anatolia », 2006; Dagmar Lorenz, op. cit., et Gotthart Wunberg, op. cit. 
autrichienne sans s'occuper du naturalisme qui n'y aurait joué aucun rôle ${ }^{7}$. Les textes critiques des écrivains et journalistes viennois de cette époque rassemblés par Gotthart Wunberg semblent pourtant prouver le contraire. Et s'il est vrai que l'Autriche avait pris, au cours du XIX siècle, un certain retard dans les différents domaines culturels, le rattrapage rapide de ce retard à la fin des années 1880 est passé par une période, certes brève mais intense, de réception du naturalisme. À y regarder de près, on pourrait même risquer l'hypothèse que la modernité viennoise est née de l'ouverture au naturalisme et des discussions que celui-ci a suscitées...

\section{La Moderne Dichtung, une revue naturaliste?}

Il est assez significatif que la première revue autrichienne qui se réclame, par son titre, de la modernité, la Moderne Dichtung, soit d'une orientation clairement naturaliste. Cette revue éditée par Eduard Michael Kafka (1868-1893) paraît d'abord, en 1890, à Brünn en Moravie, puis, pour échapper à tout provincialisme, la rédaction et l'éditeur s'installent à Vienne. Le $1^{\text {er }}$ avril 1891, la revue réapparaît sous le titre Moderne Rundschau, titre qui devait mieux signaler son ambition «d'être un miroir de toute la vie moderne », de comprendre la littérature dans le contexte de toutes les manifestations de l'esprit moderne, de comprendre les documents littéraires et artistiques du présent à la lumière des expériences scientifiques, psychologiques et sociologiques, du point de vue des opinions progressistes dans le domaine du droit, de la morale, de la technique, de l'économie et de la politique sociale ${ }^{8}$. À la fin de la deuxième année de parution, en janvier 1892, elle fusionne avec la Freie Bühne für modernes Leben éditée par Otto Brahm à Berlin.

Plusieurs facteurs montrent que la revue adhère aux idées et à l'esthétique naturalistes. Pour Eduard Michael Kafka, il s'agissait effectivement de créer une publication pour soutenir le réalisme et le naturalisme (ces deux termes n'ayant pas été séparés très clairement dans 1'esprit des critiques, le second ayant souvent été considéré comme le prolongement du premier). Elle est conçue selon le modèle de la revue Die Gesellschaft

7. Paradigmatique pour cette approche : Claude David, Von Richard Wagner zu Bertolt Brecht. Eine Geschichte der deutschen Literatur, Frankfurt/Main, S. Fischer, 1964, p. 124. Cette doxa a eu la vie longue et se trouve encore chez Jens Malte Fischer, Fin de siècle. Kommentar zu einer Epoche, München, 1978 et Iris Paetzke, Erzählen in der Wiener Moderne, Tübingen, 1992. Les travaux de Gotthart Wunberg en Allemagne et ceux d'Yves Chevrel en France (voir notamment sa thèse d'État, Le Roman et la nouvelle naturalistes français en Allemagne [1870-1893], Paris, Université de la Sorbonne, 1979 et son livre Le Naturalisme, Paris, PUF, 1982) ont cependant montré dès le début des années 1980 que cette affirmation mérite au moins d'être nuancée. Voir à ce propos aussi Gotthart Wunberg, op. cit., p. 25 et Dagmar Lorenz, op. cit., p. 47.

8. Cf. Gotthart Wunberg, op. cit., p. 23. 
(La Société), un périodique ouvertement naturaliste créé en 1885 à Munich par Michael Georg Conrad.

Un portrait de Conrad ouvre d'ailleurs le premier numéro de la revue, il est accompagné d'un article signé Arthur Gundaccar von Suttner ${ }^{9}$ consacré à cet écrivain - naturaliste - munichois, qui y est considéré comme celui qui a fait entrer dans la littérature allemande un esprit de liberté ${ }^{10}$.

Le contenu du premier numéro de la revue donne à penser que l'éditeur cherchait à établir une communauté naturaliste entre Vienne, Munich et Berlin et ne laisse aucun doute sur son orientation : on y trouve des nouvelles de M.-G. Conrad et de Timm Kröger (un collaborateur de Die Gesellschaft), des poèmes de Detlev von Liliencron et d'Arno Holz, un essai programmatique de Wilhelm Bölsche, l'un des théoriciens du naturalisme allemand, le compte rendu d'une pièce (naturaliste) de Hermann Bahr, Die große Sünde (Le Grand pêché) et des travaux de trois écrivains autrichiens classés comme « réalistes » : Ludwig Anzengruber (1839-1889), Ferdinand von Saar (1833-1906) et Leopold von Sacher-Masoch (1836-1895). Les portraits dont est doté chacun des douze numéros de la première année ainsi que les articles confirment ce constat: on y trouve, entre autres, les portraits d'Azengruber, von Saar, Gerhart Hauptmann, Georg Brandes et de Sacher-Masoch.

Mais si l'on prend en compte la composition de l'ensemble des douze numéros de la première année, on peut constater un glissement progressif qui semble éloigner la revue autrichienne de ses modèles munichois et berlinois. Dès le premier numéro (janvier 1890), la revue publie un essai de Hermann Bahr intitulé « Die Moderne » qui amorce déjà, comme nous le verrons plus tard, le dépassement du naturalisme, et on y trouve également les premiers travaux de ceux qui ne tarderont pas à être considérés comme les phares de la «modernité viennoise»: Hofmannsthal (1874-1929), Schnitzler (1862-1931), Dörmann (1870-1928) et Salten (1869-1947). Au cours de la première année se produit donc une ouverture à d'autres courants, à d'autres écrivains que les représentants d'un réalisme-naturalisme traditionnel.

Mais il semble que, dans l'euphorie des débuts, personne ne s'en aperçoit. Ainsi, Otto Julius Bierbaum, critique littéraire berlinois favorable au naturalisme, consacre dans Die Gesellschaft un compte rendu de six pages au premier numéro de Moderne Dichtung. Il y considère « ce nouvel organe du réalisme littéraire comme un beau signe du progrès de notre affaire » et sa création comme un "acte prometteur pour le réalisme allemand ». Il y exprime aussi son espoir que cette nouvelle revue saura éviter les excès qui ont fait tant de mal au naturalisme, même si ceux-là

9. Il s'agit du mari de Bertha von Suttner, ancienne secrétaire et amie d'Alfred Nobel et futur Prix Nobel de la paix (1905). Elle venait de publier, en 1889, son roman pacifiste Die Waffen nieder! (A bas les armes!).

10. Cf. Gotthart Wunberg, op. cit., p. 67. 
s'expliquent par la fougue d'une jeunesse voulant imposer une littérature nouvelle ${ }^{11}$. En réalité, la ligne éditoriale de la revue est vague, mais elle soutient la revendication naturaliste de la littérature comme étant «un miroir de la vie moderne ${ }^{12} »$.

\section{La visite d'Ibsen}

La Moderne Rundschau, qui prend la relève de la Moderne Dichtung à partir d'avril 1891, est liée à un autre événement qui confirme les débuts naturalistes de la modernité viennoise, la venue d'Ibsen à Vienne.

Max Burckhard, qui venait d'être nommé directeur du prestigieux Burgtheater, avait mis au programme de sa première saison Les Prétendants à la couronne et invité Ibsen à assister à la première le 11 avril 1891. Les éditeurs de la Moderne Rundschau, Eduard Michael Kafka et Jacques Joachim, organisèrent à cette occasion un banquet en l'honneur d'Ibsen, qui fut l'un des moments forts du séjour du dramaturge norvégien en Autriche et un grand événement politico-culturel. La Moderne Rundschau offrit aux invités un tiré-à-part comportant un portrait d'Ibsen et un essai de Julius Kulka ${ }^{13}$, dans lequel on trouve les paradigmes habituels de la réception d'Ibsen dans le monde germanique : l'opposition, dans ses pièces, de nouvelles vérités aux anciennes qui, par là, seraient démasquées comme mensonges. Quelques éléments moralisateurs dans son œuvre seraient contraires à l'esprit naturaliste, mais, comme Zola, Ibsen serait à la fois naturaliste et symboliste, deux notions qui, pour Kulka, ne semblent pas s'exclure. Tout comme Hermann Bahr, Kulka considère Ibsen comme un "individualiste» et, allant en cela plus loin que Bahr, comme un « libérateur » et le « Messie » de la littérature moderne.

À ce propos, il faut aussi noter la visite que le jeune Hofmannsthal (17 ans) a rendue alors à Ibsen, surtout parce que se rattache à cette rencontre l'un des «mythes fondateurs » de la « Jeune Vienne ». Hofmannsthal note en effet dans son Journal, qu'Ibsen lui aurait parlé de « nous autres, jeunes viennois » comme d'un « groupe de jeunes artistes dont le seul but serait la création artistique ${ }^{14} \gg$.

\section{La création de la Freie Bühne}

Pour promouvoir l'esthétique moderne - dans un sens très large du terme au théâtre, les « Jeunes Viennois » créent, suivant en cela aussi les modèles berlinois et parisien, un «théâtre libre». Comme celui d'Otto Brahm à

11. Cf. ibid, p. $20 s q$.

12. Cité d'après Gotthart Wunberg, op. cit., p. 23.

13. Journaliste viennois, né en 1865 , coéditeur de la Moderne Rundschau et correspondant viennois de la revue berlinoise Freie Bühne.

14. Cité d'après Gotthart Wunberg, op. cit., p. 40. 
Berlin, il prend le nom de «Freie Bühne ». Il s'agit d'une «association pour la littérature moderne » qui a d'abord pour but d'organiser des conférences, des soirées lectures, des représentations théâtrales et la création d'une bibliothèque... dans l'esprit du naturalisme, car «le naturalisme est le mouvement moderne par excellence à Vienne ${ }^{15} »$. Le président de l'association est un critique et journaliste originaire du Nord de l'Allemagne, Friedrich Michael Fels, qui a fait ses études à Vienne; les postes clefs sont tenus par des proches de la Moderne Rundschau (Eduard Michael Kafka, Edmund Wengraf); parmi les membres du Conseil d'administration on trouve quelques «Jeunes Viennois » comme Arthur Schnitzler et Félix Salten, ainsi que le père de Hugo von Hofmannsthal. Ibsen est nommé membre d'honneur. La presse viennoise a déduit de la composition de ce conseil qu'il s'agissait d'une « réunion d'écrivains qui s'étaient retrouvés sous le drapeau du naturalisme ${ }^{16}$ ». Visiblement, «modernité » et « naturalisme » ne font qu'un. Fels est, en effet, un partisan convaincu du naturalisme. Ainsi estime-t-il, dans des articles consacrés à «Ibsen » et à « Nos idéalistes », que « l'observation exacte de la nature est la première et la plus importante condition de l'art ». Au cours de l'année 1891 sa position semble pourtant évoluer, note Gotthart Wunberg ${ }^{17}$ et, dans un article sur le botaniste, traducteur et écrivain danois Jens-Peter Jacobsen, Fels constate que celuici est capable, à la suite d'une longue maladie qui l'a condamné à une vie recluse, d' " entendre mille sons, de voir mille couleurs, de sentir mille odeurs qu'un autre ne perçoit pas ${ }^{18}$ ». Même s'il semble estimer qu'une telle capacité relève d'un "manierisme ", il voit aussi que le naturalisme poussé à l'extrême peut conduire à un dépassement du naturalisme et à l'impressionnisme. Comme Hermann Bahr (voir ci-dessous), il constate un intérêt grandissant pour la forme au détriment du contenu. Cette évolution se précise dans l'allocution inaugurale qu'il a tenue lors de la première séance publique de la «Freie Bühne » le 29 octobre 1891 : il associe naturalisme et modernité, mais prend le terme de «naturalisme» dans une acception très large, considérant que, dans la mesure où il a l'ambition de donner, à sa manière, une image fidèle d'une tranche de la vie - ou de sa vie -, tout bon écrivain est un « naturaliste ${ }^{19} »$.

Ni la revue, ni l'association n'ont résisté à ce pluralisme programmatique un peu flou. Au moment où la revue a fusionné avec la Freie Bühne berlinoise (janvier 1892), l'association a été rebaptisée, sous l'impulsion

15. Cf. Norbert Bachleitner, «Le Théâtre Libre de Vienne », dans Philippe Baron (dir.), Le Théâtre libre d'Antoine et les théatres de recherche étrangers, Paris, L'Harmattan, coll. «Univers théâtral », 2007, p. 39-54, ici p. 40.

16. Dagmar Lorenz, op. cit., p. 51.

17. Cf. Gotthart Wunberg, op.cit, p. 51 sqq.

18. Cf. ibid., p. 52. L'article «J. P. Jacobsen» a été publié dans Moderne Rundschau, vol. $3, \mathrm{n}^{\circ} 7,1^{\text {er }}$ juillet 1891 .

19. Cf. Gotthart Wunberg, op. cit., p. 53 et p. 194. 
de Hermann Bahr, Verein für modernes Leben (Association pour la vie moderne). Ce changement de nom exprimait un changement programmatique qui se reflète également dans le choix de la pièce retenue pour la seule représentation théâtrale que l'association ait finalement organisée en mai 1892: L'Intruse de Maurice Maeterlinck. En introduction à la pièce, Bahr a tenu une conférence dans laquelle il a chanté les louanges du symbolisme comme « art des nerfs » («Nervenkunst») et avenir de la littérature. La représentation de L'Intruse semble avoir été un échec, les réactions de la presse plutôt négatives. La Neue Freie Presse du 3 mai 1892 estimait, par exemple, «qu'une pièce sur la mort sied mal à une association pour la vie moderne ${ }^{20} »$. Il n'empêche : la représentation de L'Intruse semble bien avoir marqué le moment où la «Jeune Vienne» bascule du naturalisme vers d'autres expressions contemporaines ${ }^{21}$.

Les tenants du naturalisme ne s'y sont d'ailleurs pas trompés : Friedrich Michael Fels regrette, dans le compte rendu qu'il a donné de la soirée dans le numéro de juin 1892 de la Freie Bühne de Berlin que « la "Jeune Vienne" courre courageusement et au pas de course derrière Hermann Bahr » pour considérer le naturalisme comme dépassé, et Edmund Wengraf polémique dans la Wiener Literaturzeitung contre Bahr en contestant au symbolisme toute modernité et en considérant celui-ci plutôt comme décadent, anticipant ainsi la critique conservatrice de la modernité ${ }^{22}$.

Que Hermann Bahr soit à l'origine de cet événement n'est pas un hasard. Cela prouve qu'il a su anticiper les évolutions et contribuer à propager les idées qu'il défendait grâce à son génie de communicateur.

Mais si les tenants du dépassement du naturalisme l'ont finalement emporté sur ses défenseurs, ce n'est peut-être pas uniquement parce que c'était dans l'air du temps. On peut penser que ce dépassement a été aussi l'occasion d'affirmer une position « autrichienne » et de se distinguer des naturalistes allemands qui avaient encore le vent en poupe ${ }^{23}$.

20. Cité d'après Norbert Bachleitner, op. cit., p. 41.

21. Encore qu'il faille relativiser le phénomène, car les drames que Schnitzler écrits dans ces années-là relèvent encore largement de l'esthétique naturaliste. Voir à ce propos mon article «Arthur Schnitzler et le naturalisme : Das Märchen à l'épreuve de la dramaturgie naturaliste », dans Franck Bauer et Guy Ducrey (dir.), Le Théâtre incarné. Études en hommage à Monique Dubar, Lille, UL3, coll. « Travaux et recherches », 2003, p. 65-76.

22. Cf. Dagmar Lorenz, op. cit., p. 53.

23. Dans son livre Les Intellectuels en Europe au XIXe siècle (Seuil, 1996), Christophe Charle avance, à propos des spécificités du champ littéraire viennois, une explication qui nous paraît complémentaire à celle évoquée ici : il estime que « les stratégies symboliques mises en œuvre par les écrivains novateurs (H. Bahr, H. von Hofmannsthal, A. Schnitzler, L. von Andrian, F. Salten, R. Beer-Hofmann) découlent de leur position structurellement contradictoire : à la fois dominants par leurs atouts multiples [...] et dominés parce qu'ils se trouvent placés aux marches de l'espace culturel germanique » (p. 236). 


\section{Hermann Bahr : la « modernité » comme dépassement (du naturalisme)}

Aussi controversé soit-il, Hermann Bahr (1863-1934) est un personnage incontournable dans toute tentative de cerner les différents aspects de la «modernité viennoise » et son action dépasse largement le domaine littéraire. Auteur extrêmement prolixe, ouvert à toutes les tendances, à toutes les évolutions, il est aussi l'un des plus discutés, voire contestés des écrivains viennois de cette époque ${ }^{24}$.

Né à Linz (Haute-Autriche) dans une famille appartenant à la bourgeoisie libérale, Hermann Bahr fait d'abord des études de philologie, de sciences politiques et de sciences économiques à Vienne, à Czernowitz et à Berlin. Il adhère aux idées nationalistes et pan-germanistes, avant d'être convaincu, lors de son séjour berlinois, de la nécessité de l'existence d'un État autrichien, voire de l'Empire austro-hongrois. Il s'intéresse également au marxisme et à la social-démocratie naissante. Mais lors de ses études, il se détourne progressivement de la politique et de l'économie, pour s'intéresser de plus en plus à l'art et à la littérature. Il fait la connaissance des naturalistes berlinois (notamment des frères Hart) et, à Berlin, lit Zola ${ }^{25}$ et Daudet.

C'est cependant son séjour à Paris en 1888-1889 qui sera le véritable déclencheur de ses activités ultérieures. Annette Daigger a retracé, dans un article récent, ce premier séjour de Bahr à Paris et les expériences qu'il y a faites. Elle insiste sur la découverte d'une vie artistique et intellectuelle effervescente, la découverte d'une ville dont l'ambiance excite ses « sinnliche Empfindungen » (« sensations sensuelles »). Il pense y trouver « le chemin de la modernité ${ }^{26} »$ : être « moderne » signifiant pour lui, dans un premier temps, haïr tout ce qui a déjà existé, rejeter tout modèle, toute imitation et obéir uniquement à ses propres sensations esthétiques et artistiques. Il fréquente les "Caf'conc' » et les cabarets et il semble que c'est en pensant à Aristide Bruant qu'il choisira plus tard, à Vienne, une bure de moine comme l'une de ses images de marque. Il lit Baudelaire, Barrès, Bourget, Huysmans, Maeterlinck, Catulle Mendès ${ }^{27}$

24. Une réévaluation de l'action de Bahr a été entreprise ces dernières années. Voir à ce propos notamment le volume de Jeanne Benay et Alfred Pfabigan (éds.), Hermann Bahr - für eine andere Moderne, Bern, Peter Lang, coll. «Convergences », 2004 et Dagmar Lorenz, Wiener Moderne (2007), op. cit.

25. Dans son Autoprotrait, Bahr se souvient de la lecture de Germinal dans les termes suivants : « Germinal tomba comme la foudre. Nous avions brusquement là, mis en forme, ce qui fermentait en nous. Nous avions là, semblait-il, le jugement dernier » (Hermann Bahr, Ce monsieur de Linz qui inventa Vienne (2006), op. cit., p. 65).

26. Annette Daigger, « Eine Wiener "badaud" in Paris. H. Bahr und das französische Fin de siècle », dans Jeanne Benay et Alfred Pfabigan (éds.), op. cit., p. 357-370, ici p. 361.

27. Sur les rapports entre Bahr et Mendès voir Guy Ducrey, « Hermann Bahr und die dekadente Lehre Catulle Mendès », dans Jeanne Benay et Alfred Pfabigan (éds.), op. cit., p. 325-338. 
et se familiarise avec l'approche critique pratiquée par Jules Lemaître et ses critiques impressionnistes.

L'incarnation de la modernité dans le domaine de la peinture est pour Bahr, à ce moment-là, Puvis de Chavanne. Cette préférence s'explique, selon Annette Daigger, par l'intérêt qu'il porte à la question de la forme de l'œuvre d'art... un intérêt qui concerne aussi la forme de l'œuvre littéraire et qui fera qu'il s'arrêtera un jour sur une phrase du Roman expérimental de Zola : «Une phrase bien faite est une bonne action ${ }^{28}$. » Si cette phrase frappe partiulièrement Bahr, si tous les exégètes de Bahr y font allusion, c'est parce qu'elle semble l'avoir libéré d'un mépris de la forme qu'il partageait alors avec les jeunes écrivains allemands. Ce mépris leur aurait été inspiré par les œuvres des épigones qui tenaient alors le haut du pavé dans la littérature allemande (Emmanuel Geibel, Paul Heyse, etc.) et qui avaient conduit les naturalistes allemands à négliger la question de la forme. Les propos de Zola conduisent Bahr à mettre en cause le naturalisme tel qu'il l'a connu et pratiqué lui-même à Berlin et à faire une distinction entre naturalisme allemand et naturalisme français :

\footnotetext{
Il m'apparut alors en toute clarté que nous n'avions rien compris, à Berlin, du naturalisme des Français. Nous l'interprétions dans un sens matérialiste, nous le prenions au mot, nous n'avions pas vu qu'il n'avait d'autre sens pour les Français que celui d'une réaction contre le romantisme, d'un retour à la tradition classique, même si c'était par un bien curieux détour. «Les brutes qui croient à la réalité des choses! » dit un jour Flaubert, lui aussi un grand maitre du naturalisme. Mais, justement, notre naturalisme berlinois ne jurait que par cette croyance à la réalité des choses ${ }^{29}$.
}

La question de la réalité « des choses », mais aussi de celle « des sensations » et de celle « de l'âme » seront au cœur du raisonnement qui mènera Bahr du naturalisme à la modernité. La « réalité des choses » l'occupe bien au début de sa carrière littéraire et se reflète dans deux drames écrits dans ses premières années berlinoises, Die neuen Menschen (Les Hommes nouveaux, 1887) et Die große Sünde (Le Grand pêché, 1889). Les dettes qu'il a envers le naturalisme et le besoin qu'il ressent de le dépasser se retrouvent dans une autre pièce qu'il publie à son retour de Paris à Berlin en 1890 : il s'agit de Die Mutter (La Mère), drame en trois actes qualifié de "moderne". Plus que la pièce elle-même, c'est le compte rendu que le jeune Hugo von Hofmannsthal en a donné dans le numéro du 15 avril 1891 de la Moderne Rundschau qui retient notre intérêt. Le jeune lycéen qui signe par son

28. Cette phrase se trouve dans la section « De la critique» du Roman expérimental dans un article intitulé " La Littérature obscène " paru initialement dans Le Voltaire du 31 août 1880 (Emile Zola, Euvres complètes, édition établie sous la direction de Henri Mitterand, t. 10, Paris, Tchou - Cercle du Livre précieux, 1968, p. 1375-1378, la citation p. 1376).

29. Citée d'après Hermann Bahr, Ce monsieur de Linz qui inventa Vienne, op. cit., p. 80. 
pseudonyme Loris a saisi avec une acuité étonnante les problèmes devant lesquels se trouvait Bahr, le passage du naturalisme à la modernité :

Le processus décrit, la synthèse entre la réalité brute et le raffinement lyrique, est presque un symbole de la tâche actuelle de l'art en général. Bahr lui-même a conçu le problème ainsi : à partir du zolaïsme et du romantisme, de l'élément épique de la rue et de l'élément lyrique du rêve, aboutir à la grande, la nouvelle, la mystique unité;

suit une autre référence zolienne :

La Mère passe à côté de ce grand problème de l'art, elle ne fait que l'effleurer, Bahr échoue dans La Mère pour avoir voulu en faire trop, comme Claude dans L'Euvre ${ }^{30}$.

Mais davantage que par ses propres créations littéraires, Bahr devient par ses essais critiques le porte parole de tout ce qui est « nouveau ». Les termes « moderne » et « modernité » occupent alors une place particulièrement importante dans son dispositif discursif et marquent les esprits. Il a rencontré ces termes sans doute lors de son séjour à Berlin, où, depuis le milieu des années 1880, la notion de "moderne » a servi à un groupe de jeunes auteurs à promouvoir une littérature nouvelle. Ainsi, Wilhelm Arent a intitulé, en 1884, une anthologie de poèmes contemporains Moderne Dichtercharaktere (Caractères d'écrivains modernes), Arno Holz a publié en 1885 Buch der Zeit. Lieder eines Modernen (Livre du temps. Chants d'un homme moderne) contenant un poème intitulé « Modern » et Kurt Wolff a tenu dans l'association littéraire Durch! en septembre 1886, peu de temps après sa création, une conférence sur « Die Moderne. Zur Revolution und Reformation der Literatur ». Mais alors que Wolff entend le terme de Moderne (« modernité ») comme opposition à Antike (« antiquité ») (« Notre plus grand idéal artistique n'est plus l'antiquité, mais la modernité », dit-il dans l'une des thèses publiés dans le Magazin für Literatur en 1886 et dans la Deutsche Universitätszeitung en 1887), la manière dont Bahr emploie le terme est plus vague, ne s'oppose pas uniquement à l'Antiquité, mais exprime, plus généralement, le désir de quelque chose de nouveau ${ }^{31}$.

\section{«Die Moderne » et « Die Überwindung des Naturalismus »}

Parmi ses nombreux articles qui reflètent ses expériences berlinoises et parisiennes, deux semblent se prêter pariculièrement bien à montrer le cheminement de la pensée de Bahr : «Die Moderne » qu'il publie dans le premier numéro de la Moderne Dichtung et «Die Überwindung des Naturalismus », article qui a donné le titre à un recueil publié en 1891.

30. Citée d'après Hermann Bahr, Ce monsieur de Linz qui inventa Vienne, op. cit., p. 147 sq.

31. Cf. Gotthart Wunberg, op. cit., p. 31. 
Dans son essai «Die Moderne ${ }^{32}$ » Bahr insiste surtout, dans un style ampoulé, voire pathétique, sur la nécessité pour la littérature de trouver «quelque chose de nouveau ${ }^{33}$ ». Le vocabulaire est flou, la direction incertaine, l'idée qui se précise au fil des lignes est celle d'une fusion de la réalité extérieure et la réalité intérieure ${ }^{34}$, la création d'une symbiose entre ce que Bahr entendait par naturalisme, par décadence et impressionnisme. L'élément naturaliste de la modernité est, pour Bahr, la recherche de la vérité, « la loi de la vérité ». Mais la vérité des naturalistes, de Zola et d'Ibsen aux naturalistes allemands, est une vérité extérieure, « matérialiste », objective, certifiée, dans certains cas, par des documents humains. Cette vérité devrait être complétée, voire remplacée par une vérité « comme chacun la ressent individuellement» («Die Wahrheit, wie jeder sie empfindet $\left.{ }^{35} »\right)$. Nous assistons donc à une « relativisation de la vérité objective » qui, grâce aux sensations ( «mpfindung »), grâce aux sens («Sinne»), devient une vérité subjective. Le lieu où se ferait cette symbiose entre la vérité extérieure et la vérité intérieure, explique Gotthart Wunberg ${ }^{36}$, serait l'âme. Et Bahr estime que la littérature germanophone aurait négligé le « côté psychologique », ce « besoin de psychologie » qu'il a trouvé chez Bourget ${ }^{37}$.

Logiquement, Bahr arrivera à la revendication que la littérature ne devrait plus s'intéresser exclusivement à «l'état des choses », mais aussi - et surtout - aux « états d'âme ${ }^{38}$ ».

Les essais du recueil Die Überwindung des Naturalismus, parmi lesquels l'article qui a donné le titre au volume, peuvent être considérés comme un point culminant dans la confrontation avec le naturalisme et l'ambition de le «dépasser», caractéristique de l'activité critique de Hermann Bahr dans les années 1890-1892. Même s'il constate dès le début que «le règne du naturalisme est passé, son rôle est terminé, son charme est rompu ${ }^{39} »$, même s'il estime, citant Maeterlinck, qu'il serait bien, qu'après les années où l'art a servi « de halle au marché de la vérité », il redevienne « le temple du rêve », Bahr ne condamne pas le naturalisme, mais lui trouve plutôt des justifications... Et insiste sur le fait que dépasser le naturalisme ne peut pas signifier un retour au classicisme

32. Je me refère ici à la traduction française de très larges extraits de cet article par Jean Launay dans Hermann Bahr, Ce monsieur de Linz qui inventa Vienne, op. cit., p. 111-117.

33. Ibid., p. 112-113.

34. Ibid., p. 115.

35. Citée d'après Gotthart Wunberg, op. cit., p. 31.

36. Ibid., p. 33.

37. Cf. Dagmar Lorenz, op. cit., p. 56.

38. Bahr décrit cette évolution dans son roman Die gute Schule, Berlin, 1890.

39. De très larges extraits de l'article « Die Überwindung des Naturalismus » sont traduits par Jean Launay dans Hermann Bahr, Ce monsieur de Linz qui inventa Vienne, op. cit., p. 129-134, ici p. 129. 
ou au romantisme. Il y aurait, selon Bahr, une différence entre l'art ancien et l'art nouveau : quand le classicisme est basé sur la raison et le sentiment, le romantisme sur la passion et les sens, la modernité, elle, s'attacherait aux nerfs. Ça serait donc un « romantisme nerveux », une « mystique des nerfs » qui permettrait de dépasser le naturalisme. Ce dernier aurait servi de correctif d'une déformation philosophique provoquée par l'art ancien et aurait ainsi contribué à l'accouchement de la modernité ${ }^{40}$.

Se basant sur le concept d'une triple " vérité » (réalité extérieure perception subjective - réalité de l'âme), Bahr développe une vision du dépassement du naturalisme qui se ferait en trois phases : il y aurait d'abord le naturalisme, le point de vue sur l'extérieur, décrire l'étranger, le dehors; puis il s'agirait de répondre au besoin d'une explication psychologique, de décrire comment l'homme s'est renouvelé, d'exprimer ce qui le pousse, ses désirs, ses énigmes; cette découverte de la psychologie, « les retrouvailles joyeuses avec la curiosité de soi, l'écoute du désir propre » mèneraient finalement à la troisième phase de la modernité, une phase marquée par la « révélation de soi, de l'ego intime, de l'étrange particularité, du nouveau bizarre », ce que Bahr considère comme « le niveau des nerfs ${ }^{41}$ ».

Les termes employés par Bahr correspondent à la terminologie habituelle à l'époque dans le discours philosophique et scientifique. Des notions comme « nerfs », « nervosité », « sensations », « atmosphère » font bien partie des mots clefs de la fin-de-siècle. Le « dépassement» devient pour Bahr une notion essentielle qui caractérise la modernité comprise comme un processus dynamique. Il s'agit, dans l'esprit de Bahr, d'un perpétuel remplacement d'un courant par le suivant, celui qui le dépasse. La conséquence logique est que Bahr révise régulièrement ses positions théoriques et prône, après le dépassement du naturalisme, celui du symbolisme, puis celui du néoromantisme et de la " mystique des nerfs » pour arriver à l'impressionnisme qui, à son tour, sera dépassé par l'expressionisme.

La lecture des numéros de la Moderne Dichtung/Moderne Rundschau montre que Hermann Bahr n'est évidemment pas le seul écrivain autrichien de l'époque qui ait eu une bonne connaissance de ce qui s'est passé, en terme de discussions esthétiques et de tentatives de renouvellement de la littérature, en Allemagne, en Angleterre et, particulièrement, en France. La plupart des jeunes écrivains viennois de la fin du XIX ${ }^{e}$ siècle semblent être à la recherche de la modernité. Pour eux, "moderne » signifie : changement/renversement. Il s'agit d'un processus qui ne se produit pas d'une manière isolée, mais en accord avec d'autres domaines de la vie. Si l'on admet que la modernité viennoise serait un choc («Aufeinanderprallen») de tendances modernistes et antimodernistes ${ }^{42}$, il faut préciser, à la lumière

40. Ibid., p. 132.

41. Ibid., p. 133.

42. Cf. Dagmar Lorenz, op. cit., p. 5. 
des articles de la Moderne Dichtung/Moderne Rundschau, qu'à la fin des années 1880 le naturalisme est encore considéré comme « moderne ». Son dépassement constituerait donc une étape de la modernité ${ }^{43}$.

Le cas de Hermann Bahr fournit un paradigme de l'évolution qui conduit les écrivains de la «Jeune Vienne» d'un intérêt certain pour le naturalisme à ce qu'on devait appeler l'impressionnisme et à l'esthéticisme fin-de-siècle.

Si Bahr a pu paraître comme un grand passeur d'idées, c'est grâce à son activité inlassable de publiciste. Il brasse ses idées dans de nombreux articles réunis ensuite dans des recueils. À partir de l'année 1891, quand il s'installe pour vingt ans à Vienne, il développe toute une stratégie éditoriale. Son but est de présenter les jeunes écrivains viennois, malgré leurs différences, comme un groupe homogène ouvert à la modernité occidentale et ayant des particularités régionales communes. Il compte ainsi créer une « image de marque » qui permettrait à ces écrivains d'être identifiables comme écrivains viennois / « Jeunes Viennois » dans le champ de la littérature de langue allemande et à lui-même de se présenter comme le représentant de ce groupe.

En défendant l'existence et la spécificité d'une littérature autrichienne et en mettant en avant le terme de «modernité viennoise», Bahr crée un moyen d'identification qui a aussi un but politique : montrer que Vienne et l'Autriche sont prêtes, sur le plan culturel, à relever le défi lancé par Berlin. En créant l'image d'un groupe homogène d'écrivains viennois, Bahr crée, en quelque sorte, un fait accompli, une réalité qu'il présente au public allemand. En même temps, il utilise les réactions de la presse berlinoises, les commentaires et avis des lecteurs berlinois pour imposer et légitimer ses propres choix à Vienne. Cette stratégie a été couronnée de succès : Bahr a sans doute facilité l'accès des écrivains autrichiens au marché allemand et il s'est présenté, en même temps, comme organisateur d'un groupe, qui, en réalité, n'en était pas vraiment un : tout en se rencontrant régulièrement au café, notamment au célèbre "Griensteidl», ces auteurs avaient des personnalités, des intérêts et des choix littéraires bien différents et étaient loin d'avoir un programme commun. En réalité, ils n'avaient même pas attendu Bahr pour se considérer comme les représentants d'une littérature autrichienne rajeunie, ouverts à toutes les expériences de la littérature occidentale contemporaine. Mais c'est Hermann Bahr qui en a finalement

43. Le fait que le dépassement du naturalisme soit devenu un facteur caractérisant la littérature autrichienne de la fin du XIX ${ }^{\mathrm{e}}$ siècle n'est pas sans conséquences pour la réception des œuvres des auteurs de la « Jeune Vienne ». Ainsi, la critique et l'histoire littéraire ont, par exemple, négligé les drames sociaux que Schnitzler a écrits dans les années 1890 et qui sont encore largement redevables de l'esthétique naturaliste (cf. plus haut p. 142, note 21) et ont pratiquement exclu du champ de la «Jeune Vienne» des écrivains comme Jakob Julius David, auteur de romans sociaux comme Am Wege sterben (Berlin, 1900) proche du naturalisme. 
assuré la promotion, en appliquant une stratégie de communication que l'on pourrait qualifier, elle aussi, de « moderne ${ }^{44} »$. Ou, selon les termes de Jean Launay, " interprète bruyant d'une génération ou émanation de l'air du temps, Bahr serait, de toute façon, représentatif, sinon même un représentant au sens commercial du mot, un publicitaire avant la lettre ${ }^{45} »$. Les mots modern et Modernität ( « moderne », « modernité ») apparaissent alors effectivement comme des Kampfbegriffe (des « notions de combat») qui servent à celui qui les emploie à imposer ses formes d'écriture préférées et à dénoncer d'autres positions comme arriérées et traditionnalistes ${ }^{46}$. La conséquence pour les écrivains étiquetés «Jeunes Viennois » est que leurs œuvres sont jugées, dans leur pays comme à l'étranger, à l'aulne du « dépassement du naturalisme » et que ceux qui ne semblent pas s'inscrire dans ce dépassement ne profitent pas de la réputation dont jouit la culture viennoise « fin-de-siècle ».

\author{
Karl Zieger \\ Université de Valenciennes
}

44. À ce propos, un débat a d'ailleurs éclaté, ces dernières années, dans la critique germanophone sur l'interprétation à donner aux écrits de Bahr et sur la qualification de son attitude : s'agit-il, comme le soutient depuis longtemps Gotthart Wunberg, d'une position dynamique qui fait de la "modernité » une notion de création active, ou, plutôt, d'une association d'antagonisme (internationalité versus régionalisme; modernité versus traditionalisme) dans le but de créer, à travers la notion de « modernité » une identité autrichienne, comme le prétend Gregor Streim? Sur ce débat sur la «modernité » ou le «traditionalisme » de Bahr, voir Dagmar Lorenz, op. cit., p. 59.

45. Jean Launay, «Introduction», dans Hermann Bahr, Ce monsieur de Linz qui inventa Vienne, op. cit., p. 7-17, ici p. 9.

46. Cette idée est émise par Horst Thomé dans son article «Modernität und Bewusstseinswandel in der Zeit des Naturalismus und des Fin de siècle », dans York-Gotthart Mix (dir.), Naturalismus, Fin de siècle, Expressionismus 1890-1918, München, dtv, coll. « Hansers Sozialgeschichte der deutschen Literatur », 2000, p. 15-27, ici p. 15. 